\title{
Reorganization in secondary somatosensory cortex in chronic low back pain patients
}

Hotz-Boendermaker, Sabina ; Marcar, Valentine L ; Meier, Michael L ; Boendermaker, Bart ;

Humphreys, Barry K

\begin{abstract}
STUDY DESIGN: A cross-sectional comparative study between chronic low back pain (CLBP) patients and healthy control subjects. OBJECTIVE: The aim of this study was to investigate reorganization in the sensory cortex by comparing cortical activity due to mechanosensory stimulation of the lumbar spine in CLBP patients versus a control group by using functional magnetic resonance imaging (fMRI). SUMMARY OF BACKGROUND DATA: LBP is now the number 1 condition across the world in terms of years living with a disability. There is growing evidence that maladaptive changes in the processing of sensory input by the central nervous system are central to understanding chronic (back) pain. METHODS: Nonpainful, posterior-anterior (PA) movement pressure was applied manually to lumbar vertebrae at L1, L3, and L5 in 13 healthy subjects and 13 CLBP patients. The manual pressure (30 N) was monitored and controlled using sensors. A randomized stimulation protocol was used consisting of 51 pressure stimuli of 5 seconds duration. fMRI data analysis was performed for the group activation within the primary and secondary sensory cortices (S1 and S2, respectively) and the representation of the individual vertebrae was extracted and statistically analyzed. RESULTS: Nonpainful PA pressure revealed no cortical reorganization in S1. In contrast, the extent of S2 activation in the CLBP group was significantly reduced in both hemispheres. In the control group, a somatotopy was identified for the lumbar vertebrae between L1 and L3, respectively, and L5 in S2 of the right hemisphere. Most importantly, a blurring of the somatotopic representation of the lumbar spine in $\mathrm{S} 2$ was observed in the patient group. CONCLUSION: Together, these maladaptive changes suggest a reorganization of higher-order processing for sensory information in CLBP patients that might have implications for a decreased sensory acuity, also related to body perception and subsequent altered functioning of the lumbar spine. LEVEL OF EVIDENCE: 2 .
\end{abstract}

DOI: https://doi.org/10.1097/BRS.0000000000001348

Posted at the Zurich Open Repository and Archive, University of Zurich

ZORA URL: https://doi.org/10.5167/uzh-135242

Journal Article

Published Version

Originally published at:

Hotz-Boendermaker, Sabina; Marcar, Valentine L; Meier, Michael L; Boendermaker, Bart; Humphreys, Barry K (2016). Reorganization in secondary somatosensory cortex in chronic low back pain patients. Spine, 41(11):667-673.

DOI: https://doi.org/10.1097/BRS.0000000000001348 


\title{
Reorganization in Secondary Somatosensory Cortex in Chronic Low Back Pain Patients
}

\author{
Sabina Hotz-Boendermaker, PhD, Valentine L. Marcar, D.Phil, Michael L. Meier, PhD, \\ Bart Boendermaker, MSc, and Barry K. Humphreys, PhD
}

Study Design. A cross-sectional comparative study between chronic low back pain (CLBP) patients and healthy control subjects.

Objective. The aim of this study was to investigate reorganization in the sensory cortex by comparing cortical activity due to mechanosensory stimulation of the lumbar spine in CLBP patients versus a control group by using functional magnetic resonance imaging ( $\mathrm{fMRI}$ ).

Summary of Background Data. LBP is now the number 1 condition across the world in terms of years living with a disability. There is growing evidence that maladaptive changes in the processing of sensory input by the central nervous system are central to understanding chronic (back) pain.

Methods. Nonpainful, posterior-anterior (PA) movement pressure was applied manually to lumbar vertebrae at L1, L3, and L5 in 13 healthy subjects and 13 CLBP patients. The manual pressure $(30 \mathrm{~N})$ was monitored and controlled using sensors. A randomized stimulation protocol was used consisting of 51 pressure stimuli of 5 seconds duration. fMRI data analysis was performed for the group activation within the primary and secondary sensory cortices (S1 and S2, respectively) and the representation of the individual vertebrae was extracted and statistically analyzed.

Results. Nonpainful PA pressure revealed no cortical reorganization in S1. In contrast, the extent of S2 activation in the CLBP group was significantly reduced in both hemispheres. In the control group, a somatotopy was identified for the lumbar vertebrae between L1 and L3, respectively, and L5 in S2 of the

From the Chiropractic Department, University of Zurich and University Hospital Balgrist, Zurich, Switzerland.

Acknowledgment date: June 30, 2015. First revision date: October 2, 2015. Acceptance date: October 27, 2015.

The manuscript submitted does not contain information about medical device(s)/drug(s).

The Foundation for the Education of Chiropractors in Switzerland and the Balgrist Foundation in Switzerland grant funds were received in support of this work.

No relevant financial activities outside the submitted work.

Address correspondence and reprint requests to Sabina Hotz-Boendermaker, PhD, University Hospital Balgrist, Forchstrasse 340, 8008 Zurich, Switzerland; E-mail: shotz@isr.balgrist.ch

DOI: 10.1097/BRS.0000000000001348 right hemisphere. Most importantly, a blurring of the somatotopic representation of the lumbar spine in S2 was observed in the patient group.

Conclusion. Together, these maladaptive changes suggest a reorganization of higher-order processing for sensory information in CLBP patients that might have implications for a decreased sensory acuity, also related to body perception and subsequent altered functioning of the lumbar spine.

Key words: body schema, chronic pain, fMRI, low back pain, manual therapy, postural control, reorganization, somatosensory cortex, spinal stimulation.

Level of Evidence: 2

Spine 2016;41:E667-E673

ow back pain (LBP) is now the number 1 condition across the world in terms of years living with a disability $^{1}$ with lifetime prevalence as high as $85 \%$. Best estimates suggest that the prevalence of chronic LBP (CLBP) is about $23 \%$, with 11 to $12 \%$ of the population being disabled by it. ${ }^{2}$ In spite of its high incidence and lifetime prevalence, there is poor evidence on the precise origins of this disorder. Accumulating evidence suggests that in the chronic condition, treatments based on structural abnormalities of patients in the musculoskeletal system are frequently unsuccessful, suggesting that pathological bio-psychosocial mechanisms are involved beside end-organ dysfunction. ${ }^{3}$ On the behavioral side, there is increasing evidence of sensorimotor malfunctions in patients with CLBP, related to chronic symptoms that might eventually result in disability. ${ }^{4,5}$ Still, the underlying cortical processing of sensory information of the lower back has not been investigated in detail in (C)LBP patients. In healthy individuals, we have recently reported cortical processing of a nonpainful manual technique, that is, posterior-to-anterior (PA) pressure onto lumbar vertebrae. ${ }^{6,7}$ Strong and consistent activation were detected bilaterally in the somatosensory cortices by using functional magnetic resonance imaging (fMRI). Between individual subjects, the activation maps in the primary somatosensory cortex (S1) were remarkably robust, although individual lumbar vertebrae were not somatotopically represented. The application of this clinically administered technique in CLBP patients might be 
helpful in disentangling maladaptive sensory processing of mechanosensory information that forms the basis for functioning of the spine. In earlier investigations in CLBP patients, brain activity induced by painful intracutaneous electric stimuli revealed a stronger and medially shifted cortical activity in $\mathrm{S} 1$ than the control group. ${ }^{8}$ In addition, an fMRI study showed a similar result on the basis of unpleasant bilateral mechanical stimulation of the lower back and the reorganization within S1 was related to the emotional impact of CLBP. ${ }^{9}$ Common to these investigations was the focus on the processing of painful stimuli. In contrast, the present fMRI study aims to investigate the organization of the sensory system related to nonpainful stimulation of the lower back in healthy subjects and possible neuroplastic changes in CLBP patients. These findings may broaden the basis for the understanding of clinical malfunctions in CLBP patients and in the future might contribute to develop novel approaches for manual therapy interventions.

\section{MATERIALS AND METHODS}

\section{Participants}

Thirteen right-handed healthy subjects and 13 CLBP patients participated in this fMRI study. Groups were age (two-sample $t$ test, $P=0.14$ ) and sex-matched (Chisquare test, $P=0.12$ ). CLBP patients were recruited from private physiotherapy practices. Participants with CLBP were included, if they had nonspecific LBP that persisted for longer than 6 months. LBP was experienced in the lumbosacral region, but in some patients, pain also radiated to the gluteal region and the upper legs. Demographic and clinical characteristics are displayed in Table 1, although information on pain duration and employment status were not collected. Exclusion criteria for control subjects were LBP within the last 6 months and a history of chronic pain. None of the participants had a history of vascular, neurological, or psychiatric illness. The study protocol was approved by the Ethics Committee of the Canton Zurich (KEK-ZH 2012-0029) and was conducted in compliance with the declaration of Helsinki. All participants signed an informed consent form and were compensated for travel expenses.

\section{Experimental Procedures}

The fMRI experimental procedures are described in detail in the study by Boendermaker et al. ${ }^{6}$ Before the fMRI experiment, the participant's lumbar vertebrae L1, L3, and L5 were palpated and their location marked on the skin. The interleaved spinous processes were selected on the basis of the segmental overlapping innervations of neighboring vertebrae. $^{10,11}$

All participants were in the prone position in the magnetic resonance (MR) scanner. The mechanical stimulation consisted of a nonpainful PA pressure exerted by the same experienced manual therapist (Author BB), with a reinforced thumb grip onto the spinous processes of L1, L3, and L5. The pressure force was $30 \mathrm{~N}$ and applied to provoke a minor PA intervertebral shear stress at the beginning of the passive range of motion that is relatively free of resistance and adverse effects. ${ }^{12}$ To ensure equal pressure, sensors (FlexiForce ${ }^{\mathbb{R}}$ Sensors, Tekscan, South Boston, MA, USA) were attached to the previously marked spinous processes and registered the pressure force. During the stimulation, visual feedback on the applied pressure force was presented on a screen in front of the experimenter in the MR room. In addition, start and stop of the stimulation were also indicated visually by this projection. The event-related fMRI experiment consisted of PA stimulations of $5 \mathrm{~s}$ duration, randomly applied 17 times on each lumbar level and, a randomized inter-stimulus interval (ISI) of 6 to $8 \mathrm{~s}$.

\section{Data Acquisition and Analysis}

A blood oxygen level dependent (BOLD), sensitive, singleshot gradient, echo planar imaging sequence was used to acquire 32 axial slices on a 3-T MR-scanner (Philips Achieva, Best, The Netherlands). Parameters were as follows: echo time $=30 \mathrm{~ms}$, flip angle $=75$ degrees, repetition time $=2600 \mathrm{~ms}$, slice thickness $=4 \mathrm{~mm}$, inter-slice gap $=0 \mathrm{~mm}$, field of view $=220 \mathrm{~mm}$, and a matrix size of $128 \times 128$ pixels, resulting in a voxel size of $1.72 \times 1.72 \mathrm{~mm}$. A single run with 255 functional images was performed and the complete scanning phase lasted about $15 \mathrm{~min}$. SPM8 (http://www.fil.ion.ucl.ac.uk/spm) was used to perform standard spatial pre-processing (realignment, normalization, and smoothing with an $8 \mathrm{~mm}$ full-width at half-maximum isotropic Gaussian kernel). A general linear model,

\section{TABLE 1. Demographic and Clinical Characteristics for the Study Participants}

\begin{tabular}{|c|c|c|c|}
\hline \multirow[b]{2}{*}{ Variable } & \multicolumn{3}{|c|}{ Group (Mean, SD) } \\
\hline & $\begin{array}{c}\text { Chronic Low Back Pain } \\
\text { Patients } N=13\end{array}$ & $\begin{array}{l}\text { Healthy Controls } \\
\qquad N=13\end{array}$ & $\boldsymbol{P}$ \\
\hline Sex $(M / F)$ & $8 / 5$ & $9 / 5$ & 0.12 \\
\hline Age $(y r)$ & 39 (15) & $42(18)$ & 0.14 \\
\hline Pain intensity ${ }^{\dagger}$ & $5(2)$ & & \\
\hline $\mathrm{ODI}^{*}$ & $21(15)$ & & \\
\hline \multicolumn{4}{|c|}{$\begin{array}{l}\text { ODI indicates Oswestry Disability Index. } \\
{ }^{*} \text { Cerman version of the Oswestry Disability Index. } \\
\text { †Pain intensity was measured on a numeric rating scale (NRS). }\end{array}$} \\
\hline
\end{tabular}


using the hemodynamic response function, was used in the first-level analysis. To control for head movements, individual movement parameters were implemented in the firstlevel model as regressors of no interest. Statistical parametric maps were then calculated, yielding beta estimates of the model fit for each subject and condition.

\section{Group Analysis}

A random effect model was used in a second-level analysis to reveal the following group activations for pooled PA pressure of L1, L3, and L5: one-sample $t$ tests for the control and the CLBP group and two-sample $t$ tests for the contrasts control > CLBP group and vice versa. As activation was expected bilaterally in S1 and S2, regions of interest (ROIs) were defined using the probabilistic Juelich Histological Atlas (included in FSLview version 3.1: http://www.fmrib.ox.ac.uk/fsl/). Each ROI was implemented as a mask with the probability for belonging to the selected structure set to $P>0.25$. The ROI analysis for $\mathrm{S} 1$ comprised the probability maps in the medial portion of the hemispheric central region, including the mesial wall and the subareas $\mathrm{BA} 3 \mathrm{a}$, BA3b, BA1, and BA2, as described in the study by Eickhoff et $a l,{ }^{13}$ and for S2 in the parietal opercular region (OP) covering the subregions OP1, OP2, OP3, and OP4. ${ }^{13-15}$ Statistical map threshold was set at $P<0.05$ and corrected for multiple comparisons (family-wise error correction, FWE). Estimation of statistical power was not possible due to a lack of prior data. We thus estimated that our sample size would offer sufficient power for identification of meaningful signal changes evoked by lumbar stimulations, which indeed was observed.

\section{Identification of the Cortical Representation of a Single Vertebra}

For the identification of the cortical representation of a specific vertebra, we generated a functional ROI for S1 of the right hemisphere ( $\mathrm{S} 1$ of the left hemisphere yielded no activation) and bilateral S2, based on an F-Contrast $(P<0.001$, uncorrected $)$ including the pooled L1, L3, and L5 stimulations of the control and the CLBP group. We determined the functional location (MNI x, y, and $\mathrm{z}$ coordinates) of each vertebra within each ROI by calculating the center of mass (COM) of the activated voxels associated. ${ }^{16}$ Subsequently, the mean and standard deviation was computed for each participant for L1, L3, and L5. To compare the different COMs, a repeated-measures analysis of variance (ANOVA) was computed, with within-subject factors "COORDINATES" ( $\mathrm{x}, \mathrm{y}, \mathrm{z})$ and "LUMBAR LEVEL" (L1, L3, L5) and between-subject factor "GROUP" (controls, CLBP patients) using the software SPSS 20. Posthoc twosample $t$ tests and paired $t$ tests were calculated. The threshold for significance was set at $P<0.05$.

\section{RESULTS}

\section{Group Analysis}

The application of PA pressure on the lumbar spine resulted in a significant hemodynamic response in sensory cortices in the control and CLBP group. In the control group, the whole-head analysis of the pooled stimulation yielded a significant activation cluster in the right hemisphere in S1 (Figure 1, Table 2). In the CLBP group, we found activation in the right S1 (Figure 1, Table 2). Within S1, two-sample $t$ tests did not show significant group differences on the whole-head and ROI level.

PA pressure led to bilateral activations in S2 of the control group. Similar to the activation pattern seen in S1, activation in S2 was larger in the right than in the left side. The CLBP group displayed activation in the right hemisphere only (Figure 1, Table 2). Between-group differences bear an enhanced activation in the right hemisphere in S2 (MNI coordinates: $46,-12,-16, t=3.57$ ) and in the left hemisphere (MNI coordinates: $-48,-32,8, \mathrm{t}=3.37$ ) in the contrast control > CLBP group, as displayed in Figure 1.

\section{Identification of the Cortical Representation of a Single Vertebra}

In both groups, the COM showed a low cortical intersubject variability within the different vertebral levels (Table 3, Figure 2). A subsequent repeated measure

Figure 1. Pooled cortical activation in S1 and S2 as induced by PA stimulation of the lumbar vertebrae. A, Top row shows the coronal sections bottom row axial sections, and location of the slices is shown by the blue lines in the rightmost figure. The control group is displayed in red and the CLBP group in green. B, Differences between the control group and the CLBP group $(p<0.001$, uncorr).

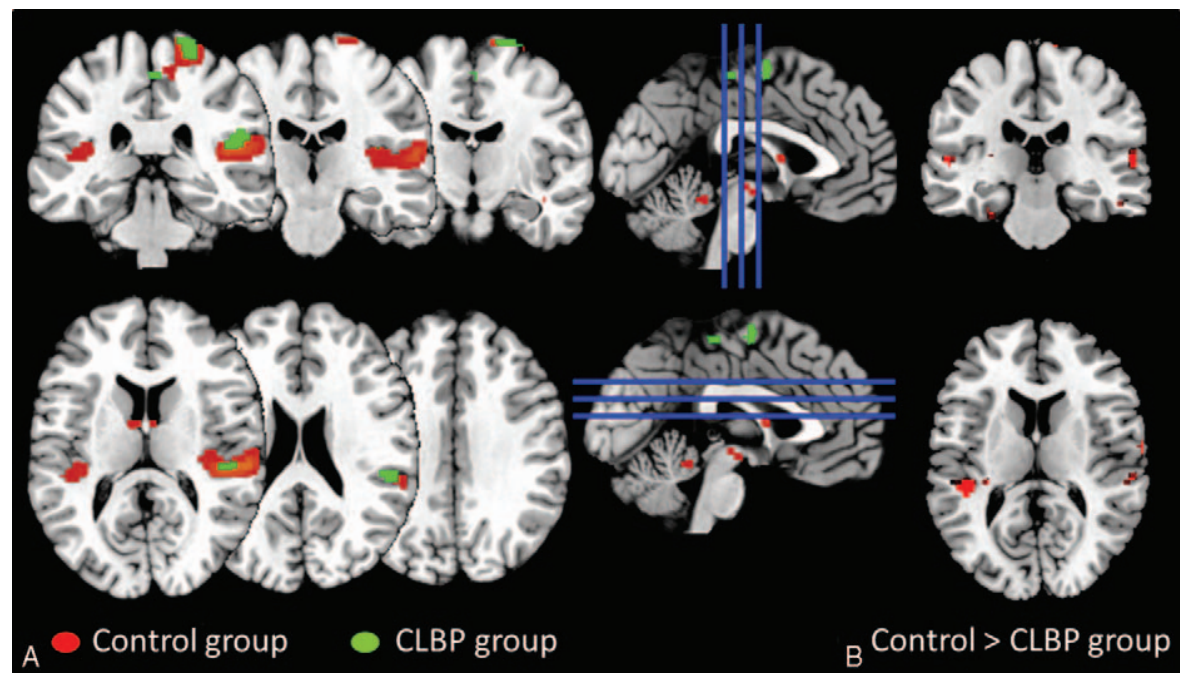




\begin{tabular}{|c|c|c|c|c|c|c|c|c|c|}
\hline & \multicolumn{6}{|c|}{ Controls $N=13$} & \multirow{2}{*}{\multicolumn{3}{|c|}{$\begin{array}{c}\text { CLBP Patients } N=13 \\
\text { Right Hemisphere }\end{array}$}} \\
\hline & \multicolumn{3}{|c|}{ Left Hemisphere } & \multicolumn{3}{|c|}{ Right Hemisphere } & & & \\
\hline & $x, y, z$ & $t$-value & $\begin{array}{c}\text { Cluster } \\
\text { Size }\end{array}$ & $x, y, z$ & $t$-value & $\begin{array}{c}\text { Cluster } \\
\text { Size }\end{array}$ & $x, y, z$ & $t$-value & $\begin{array}{c}\text { Cluster } \\
\text { Size }\end{array}$ \\
\hline S1 & & & & $18,-32,64$ & 6.64 & 416 & $20,-34,68$ & 4.24 & 83 \\
\hline S2 & $-46,-32,12$ & 5.84 & 444 & $46,-30,14$ & 5.36 & 1125 & & & \\
\hline
\end{tabular}

ANOVA of the COMs in right S1 and left S2 did not indicate significant within or between-group differences. However, in the right S2, a significant main effect of "GROUP" $(\mathrm{df}=2, F=3.19, P<0.05)$ was found. The posthoc $t$ test revealed an exclusive between-group difference for the $\mathrm{z}^{-}$ axis at $\mathrm{L} 3(\mathrm{df}=24, t=-2.48, P=0.02)$. Finally, a clear somatotopy could be identified within the right $\mathrm{S} 2$ in the control group, using paired $t$ tests. Significant differences for the $\mathrm{x}$-axes between $\mathrm{L} 1$ and $\mathrm{L} 3(\mathrm{df}=12, t=2.87, P=0.014)$ and between $\mathrm{L} 1$ and $\mathrm{L} 5(\mathrm{df}=12, t=-2.56, P=0.025)$, for the $\mathrm{y}$-axis between $\mathrm{L} 1$ and $\mathrm{L} 3(\mathrm{df}=12, t=-2.48, P=0.029)$, as well as between $\mathrm{L} 1$ and $\mathrm{L} 5(\mathrm{df}=12, t=-2.36, P=0.036)$ and along the $\mathrm{z}$-axis between $\mathrm{L} 1$ and $\mathrm{L} 3(\mathrm{df}=12, t=-2.62$, $P=0.022$ ) were detected. There were no significant differences between L3 and L5 (all $P>0.05$ ).

\section{DISCUSSION}

The study findings support the view that maladaptive processing of sensory input in the central nervous system (CNS) is central to understanding chronic (back) pain, ${ }^{17,18}$ the number 1 condition in the world in terms of years living with a disability. ${ }^{1}$ Nonpainful PA pressure revealed no cortical reorganization in S1 but reduced neuronal activity and maladaptive changes in the somatotopic representation of the lumbar spine in S2 in the CLBP group. Together, these findings strongly suggest reorganization in higher-order processing of sensory information in CLBP patients that might have implications for a decreased sensory acuity, also related to body perception and subsequent functioning of the lumbar spine.

PA pressure on spinous processes provoked motion between lumbar vertebrae that activated mechanosensors within the spines musculoskeletal structures and the skin. ${ }^{6,19}$ Such sensory information is the basis for body perception and mandatory for neural control of the lumbar spine. ${ }^{6,7}$ The present investigation confirmed that PA pressure induced a consistent activation pattern in the right $\mathrm{S} 1$ and indicated a similar representation of mechanosensory input of the lumbar spine in S1 in both the control and the CLBP group. The right hemispheric preference for processing mechanosensory spinal input is not a novel finding. ${ }^{6,9} \mathrm{~A}$ right hemisphere based network that supports the bilateral representation of the body has been discovered in studies involving patients with traumatic brain injury. ${ }^{20}$

Previous neuroimaging evidence reported reorganization in S1 related to pain intensity after the application of painful electrical stimulation in CLBP patients. ${ }^{8}$ Similarly, unpleasant mechanical vibration of the lower back demonstrated a shift of right $\mathrm{S} 1$ activation. ${ }^{9}$ These differences in our findings of similar S1 activation for nonpainful and the reorganization of $\mathrm{S} 1$ in relation with painful stimulation in previous investigations ${ }^{8,9}$ might be explained by the modality specific representation in S1. Different sensory stimuli activate distinct regions in S1. This in turn means that one sensory

TABLE 3. For the Control and the CLBP Group, Mean ( \pm Standard Deviation) MNI Coordinates for the Center of Mass (COM) in the Right S1 and bilaterally in S2 for L1, L3, and L5

\begin{tabular}{|c|c|c|c|c|c|c|c|c|c|c|c|c|}
\hline \multirow[b]{3}{*}{$\mathrm{S} 1$} & \multicolumn{4}{|c|}{ Controls $N=13$} & & & \multicolumn{4}{|c|}{ CLBP Patients $N=13$} & & \\
\hline & \multicolumn{3}{|c|}{ Left Hemisphere } & \multicolumn{3}{|c|}{ Right Hemisphere } & \multicolumn{3}{|c|}{ Left Hemisphere } & \multicolumn{3}{|c|}{ Right Hemisphere } \\
\hline & $x$ & $\mathrm{Y}$ & z & $x$ & Y & z & $x$ & Y & z & $x$ & $\mathrm{Y}$ & z \\
\hline L1 & & & & $18.8(0.22)$ & $-34.0(0.84)$ & $65.9(1.79)$ & & & & $18.9(0.55)$ & $-34.1(1.25)$ & $65.6(0.84)$ \\
\hline $\mathrm{L} 3$ & & & & $18.6(0.31)$ & -34.0. (0.66) & $65.6(1.61)$ & & & & $18.9(0.33)$ & $-34.0(0.47)$ & $65.4(0.73)$ \\
\hline $\mathrm{L} 5$ & & & & $18.82(0.73)$ & $-33.9(0.26)$ & $65.7(1.37)$ & & & & $18.60(0.37)$ & $-34.2(1.76)$ & $65.4(2.51)$ \\
\hline \multicolumn{13}{|l|}{ S2 } \\
\hline $\mathrm{L} 1$ & $-42.8(0.83)$ & $-32.1(0.45)$ & $12.3(0.89)$ & $48.5(2.03)$ & $-26.9(0.91)$ & $12.90(1.50)$ & $-43.0(2.49)$ & $-32.1(1.34)$ & $12.1(1.71)$ & $47.7(2.48)$ & $-26.9(1.19)$ & $13.9(1.54)$ \\
\hline L3 & $-42.9(0.43)$ & $-32.3(0.81)$ & $11.9(1.18)$ & $46.7(1.40)$ & $-26.4(0.81)$ & $12.8(1.62)$ & $-42.2(1.51)$ & $-31.8(1.23)$ & $12.3(1.97)$ & $47.8(1.87)$ & $-26.9(0.88)$ & $14.2(1.23)$ \\
\hline L5 & $-42.8(0.16)$ & $-31.9(0.51)$ & $11.7(0.81)$ & $46.8(2.23)$ & $\mid-26.6(0.84)$ & $12.9(1.51)$ & $-42.2(2.13)$ & $-26.9(17.5)$ & $12.4(1.40)$ & $47.4(3.08)$ & $-26.8(0.73)$ & $13.9(1.54)$ \\
\hline
\end{tabular}



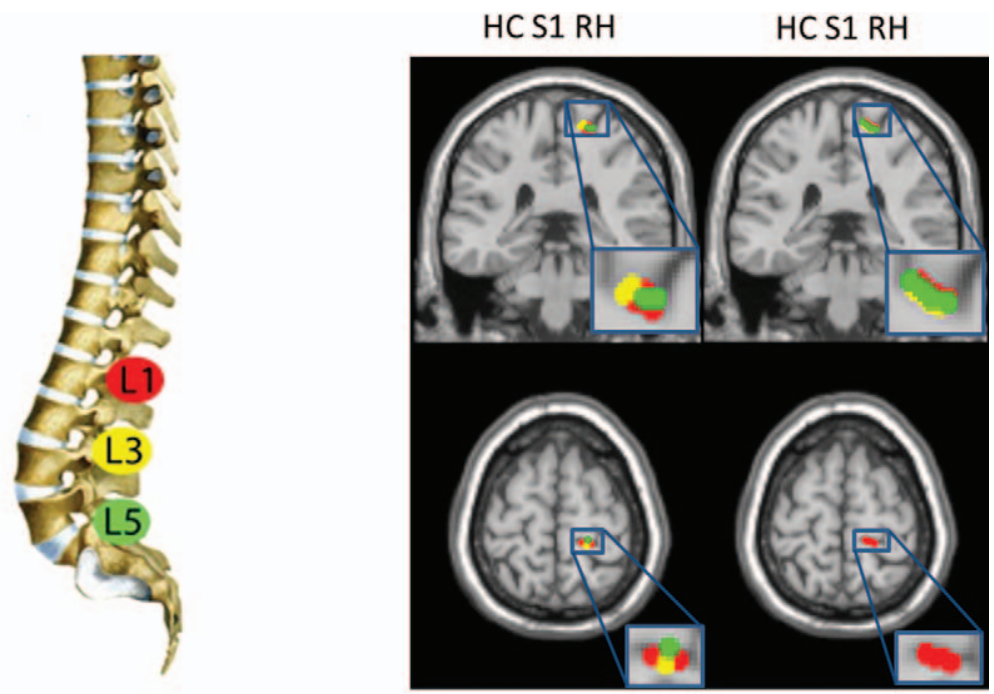

Figure 2. The top left panel depicts the location of the three sites of stimulation on the spine. The top right panel depicts the center of mass (COM) in the right cerebral hemisphere observed in S1 of the control group (left) and the CLBP patients (right). The bottom left panel depicts the COMs in the left and right cerebral hemisphere observed in S2 of the control group. The bottom right panel depicts the COMs in the left and right cerebral hemisphere observed in S2 of the CLBP patients.
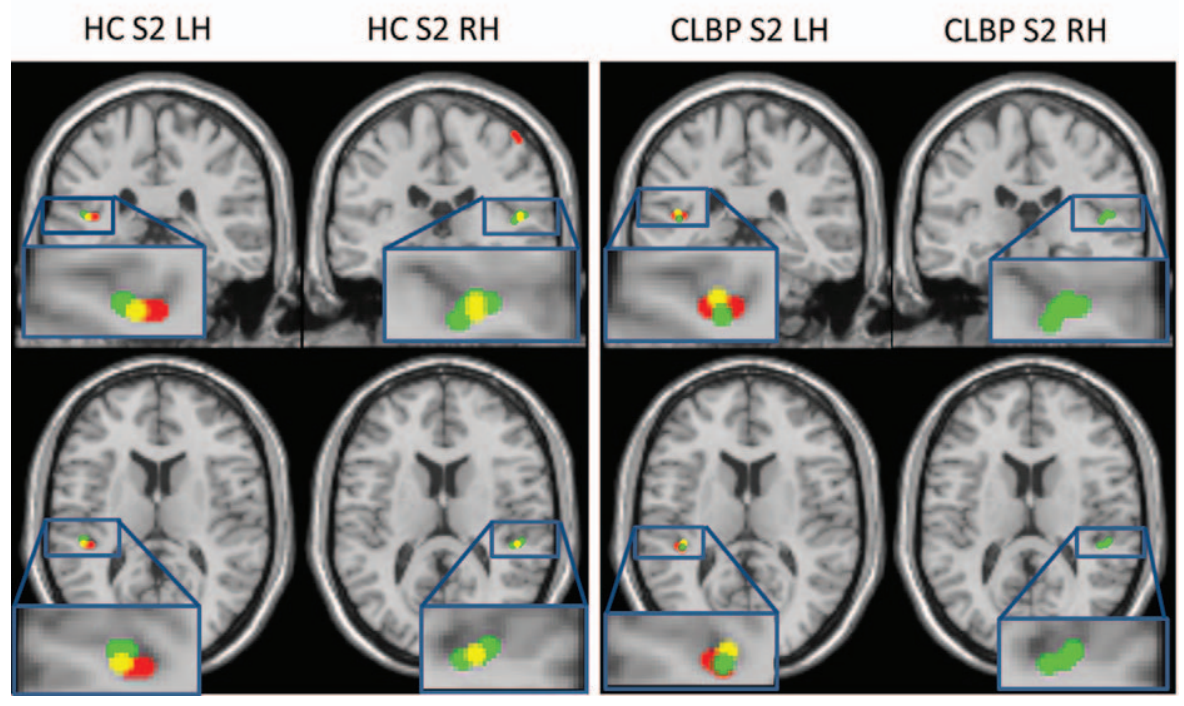

aspect (i.e., touch or pressure) tends to dominate the input in a column (i.e., vertical brain area) that represents a neuronal population, consisting of neurons of the same type. ${ }^{20}$ Recent fMRI investigations indicated a co-representation of mechanosensory and painful stimuli from the same body part in $S 1^{21}$ and both modalities were represented in a topographic order. $^{22}$ It is most probable that the pain experience draws attention to painful stimuli, as they are potentially lifethreatening, ${ }^{23}$ and results in enhanced cortical activation, a neural correlate of reorganization, such as augmented central pain processing in CLBP patients. ${ }^{24}$

In contrast to the similar strength of activation of the control and CLBP group within S1, different activation patterns in S2 of the two groups indicate a downregulation of sensory processing of this area in the CLBP group. Such a finding has not been reported for CLBP patients but has been observed in other chronic pain states such as fibromyalgia, chronic regional pain syndrome, and trigeminal neuralgia. ${ }^{15,25-27}$ Slowed recovery and high pain intensities in (C)LBP patients have been described to enhance central neuronal responsiveness to peripheral input. This central sensitization includes altered sensory processing in ascending and descending pain networks, as well as in mechanosensory processing. ${ }^{5}$ The decrease in hemodynamic responses after nonpainful lumbar spine stimulation might be based on previous interactions of painful and nonpainful inputs in sensory areas in CLBP in daily life, resulting in enhanced activation level of neurons responding to nociceptive inputs. ${ }^{21,23}$ As a consequence, central reorganization might occur, as nonpainful processing in adjacent regions might be limited and thus result in reduced sensory activation. $^{25}$ In fact, there is evidence for a thinning of the cortical thickness bilaterally in S1 and S2 in CLBP patients compared with a control group. ${ }^{28,29}$

\section{Somatotopy of the Lumbar Spine in S2}

The representation of the lower back in S1 lacks a somatotopic representation of the individual vertebrae. ${ }^{6}$ Potential 
explanations for this observation may be overlapping representation of individual vertebrae or to coarse a spatial resolution of the fMRI for mapping single vertebrae representations. ${ }^{6}$ Such a somatotopy for the lumbar spine was discovered in the right hemisphere in S2 of the control group. This was surprising, as the topographic representation in S2 has been shown to be coarser than in S1, as demonstrated by the lack of a representations of individual fingers in its hand area. ${ }^{30}$ This difference in findings may be attributable to the difference in stimulation paradigm; although the present investigation involved multimodal processing in $\mathrm{S} 2$, others used unimodal stimulation. Although sensory modalities are processed individually in S1, S2 integrates bilateral multiple somatic input and enhances attention toward sensory stimulation. This integration of different proprioceptive and tactile information might reflect the functional nature of PA pressure and result in the individual representation of lumbar vertebrae. ${ }^{31}$ Interestingly, the individual COM measures showed different locations for L1 and L3, but no difference between L3 and L5. A potential explanation for this specific topographic arrangement may mirror the difference in functional properties of L1 compared with L3 and L5. The latter are restricted in their rotation movement, as they have a predominantly weight-bearing role in the spine.

Most interestingly was the absence of this topographic arrangement of the individual vertebrae in the CLBP group, indicating a blurred representation of the lumbar spine in S2. These findings support accumulating evidence from behavioral studies that demonstrated impaired body perception in CLBP patients, ${ }^{4}$ with deficits in proprioception, ${ }^{32,33}$ disruption of vibrotactile stimuli processing, ${ }^{34}$ and poorer tactile acuity. ${ }^{35}$

Due to the complex relationship between perception and action, a disturbed lumbar spine representation may have important consequences, as sensory information is mandatory to select appropriate neural control strategies for stabilization and movement of the spine. ${ }^{19}$ Central in this process is the $\mathrm{S} 2$ that has close connections with premotor planning areas. ${ }^{36}$ Subsequently, cortical reorganization of the sensorimotor system may lead to maladaptive functional changes that might include impoverished motor programming, planning, and impaired postural control. ${ }^{37-40}$ As we have not assessed postural control in the present patient population, we are unable to further discuss these clinical appearances.

\section{Clinical Implication}

Our study has demonstrated maladaptive changes in sensory information processing and the cortical representation of the lower back in CLBP patients. Findings from our study provide an indication of the mechanism by which long-term pain leads to functional disability. Fortunately, maladaptive reorganization appears to be reversible. ${ }^{29,41}$ Therefore, research is needed to determine whether novel therapeutic approaches such as repeated, nonpainful manual input are beneficial in activation of the mechanosensory cortices and results in the restoration of normal sensory activity of the lower back and contributes to alleviating pain levels and disability in CLBP patients.

\section{Key Points}

- Maladaptive changes in sensory information processing and the cortical representation of the lumbar spine were revealed in CLBP patients.

Alterations in the sensory system may have a profound influence on the functioning of the spine by affecting body perception.

口 Findings suggest an indication of the mechanism by which long-term pain leads to functional disability.

- Maladaptive reorganization is reversible. Treatments approaches using non-painful sensorimotor stimulations could be applied to restore normal somatosensory activation of the lower back.

\section{Acknowledgment}

We thank all volunteers for their participation in the current study.

\section{References}

1. Hoy D, March L, Brooks P, et al. Measuring the global burden of low back pain. Best Pract Res Clin Rheumatol 2010;24:155-65.

2. Andersson GB. Low back pain. J Rehabil Res Dev 1997;34:ix-ix.

3. Rubinstein SM, Terwee CB, Assendelft WJ, et al. Spinal manipulative therapy for acute low back pain: an update of the cochrane review. Spine (Phila Pa 1976) 2013;38:E158-77.

4. Wand BM, Parkitny L, O'Connell NE, et al. Cortical changes in chronic low back pain: current state of the art and implications for clinical practice. Man Ther 2011;16:15-20.

5. Roussel NA, Nijs J, Meeus M, et al. Central sensitization and altered central pain processing in chronic low back pain: fact or myth? Clin J Pain 2013;29:625-38.

6. Boendermaker B, Meier ML, Luechinger R, et al. The cortical and cerebellar representation of the lumbar spine. Hum Brain Mapp 2014;35:3962-71.

7. Meier ML, Hotz-Boendermaker S, Boendermaker B, et al. Neural responses of posterior to anterior movement on lumbar vertebrae: a functional magnetic resonance imaging study. J Manipulative Physiol Ther 2014;37:32-41.

8. Flor H, Braun C, Elbert T, et al. Extensive reorganization of primary somatosensory cortex in chronic back pain patients. Neurosci Lett 1997;224:5-8.

9. Lloyd D, Findlay G, Roberts N, et al. Differences in low back pain behavior are reflected in the cerebral response to tactile stimulation of the lower back. Spine (Phila Pa 1976) 2008;33:1372-7.

10. Bogduk N. The innervation of the lumbar spine. Spine (Phila Pa 1976) 1983;8:286-93.

11. Bogduk N, Wilson AS, Tynan W. The human lumbar dorsal rami. J Anat Mar 1982;134 (Pt 2):383-97.

12. Snodgrass SJ, Rivett DA, Robertson VJ. Manual forces applied during posterior-to-anterior spinal mobilization: a review of the evidence. J Manipulative Physiol Ther 2006;29:316-29.

13. Eickhoff SB, Stephan KE, Mohlberg H, et al. A new SPM toolbox for combining probabilistic cytoarchitectonic maps and functional imaging data. Neuroimage 2005;25:1325-35.

14. Eickhoff SB, Grefkes C, Zilles K, et al. The somatotopic organization of cytoarchitectonic areas on the human parietal operculum. Cereb Cortex 2007;17:1800-11. 
15. Blatow M, Nennig E, Sarpaczki E, et al. Altered somatosensory processing in trigeminal neuralgia. Hum Brain Mapp 2009;30: 3495-508.

16. Plow EB, Arora P, Pline MA, et al. Within-limb somatotopy in primary motor cortex-revealed using fMRI. Cortex 2010;46: 310-21.

17. Pelletier R, Higgins J, Bourbonnais D. Is neuroplasticity in the central nervous system the missing link to our understanding of chronic musculoskeletal disorders? BMC Musculoskelet Disord $2015 ; 16: 25$

18. Wand BM, Catley MJ, Luomajoki HA, et al. Lumbar tactile acuity is near identical between sides in healthy pain-free participants. Man Ther 2014;19:504-7.

19. Gilchrist RV, Frey ME, Nadler SF. Muscular control of the lumbar spine. Pain Phys 2003;6:361-8.

20. Dijkerman HC, de Haan EH. Somatosensory processes subserving perception and action. Behav Brain Sci 2007;30:189-201; discussion 201-139.

21. Mancini F, Haggard P, Iannetti GD, et al. Fine-grained nociceptive maps in primary somatosensory cortex. J Neurosci 2012;32: 17155-62.

22. Omori S, Isose $\mathrm{S}$, Otsuru $\mathrm{N}$, et al. Somatotopic representation of pain in the primary somatosensory cortex (S1) in humans. Clin Neurophysiol 2013;124:1422-30.

23. Ohara PT, Vit JP, Jasmin L. Cortical modulation of pain. Cell Mol Life Sci 2005;62:44-52.

24. Giesecke T, Gracely RH, Grant MA, et al. Evidence of augmented central pain processing in idiopathic chronic low back pain. Arthritis Rheum 2004;50:613-23.

25. Pleger B, Ragert P, Schwenkreis P, et al. Patterns of cortical reorganization parallel impaired tactile discrimination and pain intensity in complex regional pain syndrome. Neuroimage 2006;32:503-10.

26. Maihofner C, Handwerker HO, Neundorfer B, et al. Patterns of cortical reorganization in complex regional pain syndrome. Neurology 2003;61:1707-15.

27. Lopez-Sola M, Pujol J, Wager TD, et al. Altered functional magnetic resonance imaging responses to nonpainful sensory stimulation in fibromyalgia patients. Arthritis Rheumatol 2014;66:3200-9.
28. Ung H, Brown JE, Johnson KA, et al. Multivariate classification of structural MRI data detects chronic low back pain. Cereb Cortex 2014;24:1037-44.

29. Seminowicz DA, Wideman TH, Naso L, et al. Effective treatment of chronic low back pain in humans reverses abnormal brain anatomy and function. I Neurosci 2011;31:7540-50.

30. Ruben J, Schwiemann J, Deuchert M, et al. Somatotopic organization of human secondary somatosensory cortex. Cereb Cortex 2001;11:463-73.

31. Keysers C, Kaas JH, Gazzola V. Somatosensation in social perception. Nat Rev Neurosci 2010;11:417-28.

32. Brumagne S, Janssens L, Janssens E, et al. Altered postural control in anticipation of postural instability in persons with recurrent low back pain. Gait Posture 2008;28:657-62.

33. O'Sullivan P. Diagnosis and classification of chronic low back pain disorders: maladaptive movement and motor control impairments as underlying mechanism. Man Ther 2005;10:242-55.

34. Moseley GL, Gallagher L, Gallace A. Neglect-like tactile dysfunction in chronic back pain. Neurology 2012;79:327-32.

35. Luomajoki H, Moseley GL. Tactile acuity and lumbopelvic motor control in patients with back pain and healthy controls. Br J Sports Med 2011;45:437-40.

36. Kaas JH. Evolution of somatosensory and motor cortex in primates. Anat Rec A Discov Mol Cell Evol Biol 2004;281: $1148-56$

37. van Dieen JH, Selen LP, Cholewicki J. Trunk muscle activation in low-back pain patients, an analysis of the literature. J Electromyogr Kinesiol 2003;13:333-51.

38. Schabrun SM, Elgueta-Cancino EL, Hodges PW. Smudging of the motor cortex is related to the severity of low back pain. Spine (Phila Pa 1976) 2015; [Epub ahead of print].

39. Gagne M, Schneider C. Dynamic influence of wrist flexion and extension on the intracortical inhibition of the first dorsal interosseus muscle during precision grip. Brain Res 2008;1195:77-88.

40. Hodges PW, Moseley GL. Pain and motor control of the lumbopelvic region: effect and possible mechanisms. J Electromyogr Kinesiol 2003;13:361-70.

41. Tsao H, Galea MP, Hodges PW. Driving plasticity in the motor cortex in recurrent low back pain. Eur J Pain 2010;14:832-9. 